\title{
Clientes, caciques y notables políticos: mecanismos de control electoral en la Sevilla de la restauración
}

\author{
MARÍA SIERRA ALONSO
}

\section{PLANTEAMIENTOS Y FUENTES}

El estudio de la vida política durante la Restauración revela la existencia de un fenómeno que, originándose en un contexto de relaciones de naturaleza privada, cumplió importantes funciones públicas y políticas: el favor. "Imposible concretar o definir ese Favor. No es un simple móvil inconfesable, un interés económico... Ni el acercamiento de un partidismo sectario que mueva a la lucha por el triunfo de unos hombres o de unas ideas. Ni el afecto de la sangre que imponga la preferencia. No. Porque todo eso, por mucho que sea su alcance, tiene un poder limitado. Y el Favor, tal como aquí se le entiende, no reconoce límite alguno...”, escribía en 1935 Jesús Pabón, criticando la vigencia de ese favor que él resaltaba con mayúscula ${ }^{1}$. Con estas páginas pretendemos ayudar a definir este fundamental mecanismo mediante el estudio de una de las manifestaciones políticas que adquirió durante la Restauración: su funcionamiento como instrumento clave para el control de los procesos electorales.

El análisis que presentamos en esta comunicación se articula en tomo a un eje protagonista: el notable político provincial, pieza crucial en el sistema político de la Restauración. La existencia de esta figura y el alcance de su poder se fundamentaron, en buena parte, sobre el mecanismo del favor. A través de la reciprocidad de favores el cacique provincial se relacionaba con el resto de la clase política española, y gracias a ello entraba en el círculo de beneficios derivados del pacto de la Restauración. Por otro lado, gracias al favor, conseguía organizar y mantener una clientela que le servía para sustentar tanto esa reciprocidad como el protagonismo público en su área de actuación. Amigos políticos y clientela eran, además, dos fuentes de poder que se respaldaban mutuamente ${ }^{2}$.

(1) PABONJ: Palabras en la oposición. Sevilla. 1935. p238.

(2) J.VARELA ORTEGA comenta, en este sentido, la .doble ficción. sobre la que vive el cacique. Los amigos políticos. Partidos, elecciones y caciquismo en la Restauración (1875-1900). Madrid. Alianza. 1977. p257. 
Desde la perspectiva del notable político provincial, por tanto, analizaremos la red de relaciones que le rodeaba y su concreta expresión en forma de mecanismos de control electoral. Distinguiremos dos ámbitos de relaciones: el de los samigos" que se encuentran incluidos en la clase política nacional e instalados en el poder central -espacio en el que el notable provincial se relaciona con personas en un nivel de poder superior a él-, y aquel otro que corresponde a las vinculaciones clientelares en el que se relaciona con personas en situación de dependencia e inferioridad, aunque en muy distinto grado-. Entre ambos espacios se desarrolla la vida política de un notable provincial; son dos argumentos de poder que determinan la importancia de su protagonismo político.

Así veremos (punto 1) cómo el establecimiento de relaciones amistosas con miembros de la clase política de rango nacional era algo fundamental para un cacique provincial. De ello dependía en gran manera su designación para la jefatura de uno de los partidos del turno en la provincia, el mantenimiento de su clientela particular y -lo que ahora nos interesa- el apoyo oficial en los momentos electorales a través de distintas fórmulas.

En las páginas que siguen (puntos 2,3 y 4), trataremos de mostrar cómo la estructura clientelar fue un marco social habitual y cotidiano para la mayoría de la población española durante la Restauración. La presentación ante el Estado de todo tipo de intereses, necesidades y derechos no se realizaba normalmente a través de cauces instituidos y públicos de comunicación entre el ciudadano y la administración, sino mediante la gestión personal realizada por estas figuras que tenían contactos privados y amistades en el poder central. Individuos particulares, oligarquías económicas y comunidades enteras se vieron incluidas en esta particular forma de vinculación, pues el recurso a la mediación de un patrocinador se dio tanto a nivel individual como de grupo, aunque con distintas consecuencias.

La protección concedida por un patrono, en forma de mediación ante el poder público, generaba en estos clientes un sentido de obligación, o al menos de gratitud, debido a la conciencia del trato discriminatoriamente favorable que gracias a él recibían de la administración ${ }^{3}$. Esta obligación quedaba a veces reforzada, en el caso de los notables económicos, por la concesión de otros beneficios derivados de

(3) ROMERO MAURAJ: -El caciquismo, en $H^{\circ}$ General de España y América. T XVI-2. Madrid. Rialp. 1981. pp 79-80: ....para que el cacique pueda ganarse una clientela, y mantenerla, partiendo del uso discriminante que se hace de los recursos de la administración, es preciso que sus clientes vean, sepan, que si reciben cosas que son importantes para ellos pero que no les corresponden por derecho, es porque interviene el cacique...". 
la propia capacidad de dispensar favores del patrono. La vinculación así generada era utilizada para respaldar la actuación política del gestor, entre otras formas como versátil instrumento de apoyo electoral.

La naturaleza del fenómeno que pretendemos estudiar nos remite forzosamente a fuentes documentales privadas, aunque éstas han sido completadas con fondos hemerográficos. Además de los archivos de políticos nacionales como Maura y Dato, hemos podido reconstruir el fenómeno clientelar en Sevilla a través de la consulta del archivo privado de una dinastía de importantes políticos provinciales: los Ybarra, jefes del partido conservador desde 1891 hasta 1916, miembros sobresalientes de la oligarquía económica provincial, y patronos de amplia clientela y valiosos amigos políticos.

\section{LOS AMIGOS POLITICOS}

Sistemáticamente, al llegar los momentos electorales, los caciques provinciales y locales recurrían en solicitud de ayuda a sus respectivos amigos políticos situados en el poder central, ya que la adecuada vinculación del cacique con uno o más políticos nacionales era fundamental para el control eficaz del proceso electoral en su área de actuación. La concesión de la protección oficial previa a las elecciones (con el carácter de encasillado), así como del apoyo posterior ante el organismo encargado de validar las actas; eran, por lo general, argumentos decisivos en el mundo político de la Restauración. En la base de esta relación encontramos la evidencia de un hecho fundamental: el apoyo prestado a los caciques provinciales y locales por los amigos políticos -y su concreta efectividad electoral que ahora nos interesa- no es sino una de las formulaciones básicas del amplio pacto, basado en la reciprocidad en el favor, sobre el que se sustenta el sistema político de la Restauración. El favor dispensado por el político nacional se fundamenta en una hipotética devolución del mismo, y, efectivamente, el cacique provincial no sólo funciona como simple transmisor pasivo, de favores hacia su clientela, sino que se sitúa ante el político nacional como una instancia de poder per se, que tiene algo que ofrecer a cambio. Ese algo suele ser, precisamente, su capacidad de influencia sobre una clientela y un área de actuación (provincia, distrito o pueblo) ${ }^{4}$.

Asumiendo, por tanto, este punto de partida, al proceder a la reconstrucción de esta fórmula política en un ámbito provincial como el de Sevilla, hemos empezado por concretar tal fundamento teórico en otros

(4) J.ROMERO MAURA expone esta mutua necesidad existente entre el cacique local y el político nacional en su trabajo ya citado. pp 79-84. 
más prácticos, para luego analizar su aplicación electoral. El archivo privado de la familia Ybarra ofrece la posibilidad de conocer ambas facetas, con el particular interés de referirse a un tipo de notable provincial con una importante red de amigos políticos.

Así, la documentación privada nos ha permitido reconstruir el específico fundamento de la correspondencia de favores -con posterior utilización electoral- entre un dirigente provincial como Ybarra y distintos miembros de la clase política española. La reciprocidad en el favor se basa en la capacidad de Ybarra de responder a la protección de líderes como Maura o Dato con las prestaciones de una clientela privada y política, que no sólo materializa el turno en el gobierno en actas electorales sino que puede, además, ayudar a estos líderes en su acceso a la jefatura nacional del partido. En 1913, por ejemplo, fue decisiva la actitud de Ybarra como jefe del partido conservador sevillano para decidir la adhesión del mismo a Dato como nuevo jefe nacional, en la difícil coyuntura del rompimiento maurista ${ }^{5}$.

Por otro lado, el cacique puede incluirse en este círculo de beneficios mutuos no sólo como político profesional que ofrece su capacidad de influencia sobre terceros, sino que además, si es un notable económico, puede respaldar su actuación política sobre una fuente de poder propia y exclusiva. La fortuna privada faculta a este cacique para erigirse, por sí mismo, en directo dispensador de favores ante los políticos madrileños. Es el caso de los Ybarra, a quienes varios políticos nacionales recurrieron en este sentido. Así lo constatan distintas cartas en las que se les solicitaba para recomendados la concesión de puestos de trabajo en la principal empresa de la familia, una compañía de navegación: si Dato pedía un puesto para un joven piloto, Maura apuntaba más alto al solicitar para un amigo la consignación de la compañía en Cartagena, al igual que lo hacía Sánchez de Toca para Málaga ${ }^{6}$.

De manera significativa, el cultivo de la reciprocidad en el favor no sólo se daba entre los amigos políticos del mismo partido: el pacto de la Restauración es tan amplio, que esta fórmula de vinculación afecta a caciques provinciales y figuras nacionales de distinto color político. En 1905, por ejemplo, el Ministro liberal de Gracia y Justicia, González Peña, agradecía en una carta privada a Tomás Ybarra, recién elegido senador conservador por Sevilla, el apoyo a su candidatura en las mismas elecciones: *...el resultado de nuestra elección me ha sido tanto más halagüeño

(5) SIERRA ALONSO,M: -Maura y la crisis del turnismo: la actitud del Partido Conservador sevillano., Revista de Historia Contemporánea (Sevilla), №5 (Junio), pp 157-169.

(6) Carta de Dato a Eduardo Ybarra, 7.12.1905; carta de Maura a Tomás Ybarra (1913); carta de Sánchez de Toca a Tomás Ybarra (1905). Archivo Ybarra (Sevilla). 
cuanto que usted, su hermano y buen amigo mío ly jefe provincial del partido conservador] y todos los que siguen sus inspiraciones han tenido la bondad de apoyar mi candidatura. Nunca olvidaré tan señalado favor ${ }^{7}$. En correspondencia, Ybarra estará capacitado para solicitar a políticos liberales, con posibilidades de éxito, actuaciones electorales a favor de conservadores, como luego veremos. Creemos interesante resaltar este fenómeno de reciprocidad en el favor entre políticos conservadores y liberales por considerarlo altamente revelador de la naturaleza consensuada del sistema político de la Restauración, a la vez que clara evidencia de las escasas diferencias que separaban a los dos partidos de gobierno.

Procedamos ahora al análisis de las funciones electorales concretas que adquiere el apoyo prestado por los amigos políticos al cacique provincial. Resulta evidente que las fórmulas de apoyo más importantes son aquellas que se derivan del uso del cargo público ocupado por el amigo político, pero también mencionaremos métodos de presión derivados de otro tipo de influencias. En el primer origen se sitúan dos fórmulas de control fundamentales: el *arreglo* de los ayuntamientos antes de las elecciones y la presión a favor de la aprobación o anulación de las actas discutidas después de celebrarse los comicios.

La alteración de las corporaciones municipales a favor del partido que ocupaba el poder era una de las piezas claves del engranaje electoral de la Restauración, y la sustitución de elementos opuestos por otros afine's se recubría con una apariencia de legalidad. En las fechas que precedían a las elecciones, los políticos provinciales presionaban a las instancias de poder con capacidad de activar estos procesos: por un lado, a los amigos y clientes situados en la administración de justicia (como veremos en el punto 2) y por otro, a los políticos nacionales a los que estaban vinculados. La solicitud, e incluso exigencia en algunos casos, de arreglos en los ayuntamientos movilizaba a los distintos caciques, que intensificaban en estos momentos sus viajes a Madrid o su correspondencia con los amigos políticos instalados en el poder central. Un buen ejemplo de estas pretensiones lo constituye la carta del Marqués de San Marcial, cacique conservador del sevillano distrito de Utrera, a Villaverde, entonces Ministro de Hacienda, reclamando su mediación ante el de Gobernación, Dato: tras contarle cómo éste le había prometido modificar los ayuntamientos de su distrito (.dando a mis amigos mayoría en el de Utrera y por completo el de Villafranca y Los Palacios y dejando para más adelante la renovación en los de Lebrija y Las Cabezas,), y dado que el Gobernador Civil aún no había efectuado todos los cambios ("y esto me coloca en una situación que se (7) Carta de González Peña, Ministro de Gracia y Justicia a Tomás Ybarra, 28.9.1905.
Archivo Ybarra (Sevilla). 
agrava por momentos y a mi hermano, actual alcalde de Utrera, en el triste caso de tener un serio disgusto en cada sesión.), le solicita que presione para que el Gobernador Civil ‘dé cumplimiento a lo acordado y me nombre 7 concejales en Utrera y renueve el Ayuntamiento de Villafranca que es liberal.... ${ }^{8}$. Más templado en sus peticiones solía ser Ybarra, como jefe provincial del partido y mediador entre el más primitivo y erústico caciquismo local y las necesidades electorales del Gobierno. En su correspondencia con distintos Ministros de Gobernación, los principales responsables del arreglo de ayuntamientos, se constata la moderación en el uso de este recurso. Aun así, a pesar de la predisposición al acuerdo de políticos como Ybarra, hay casos en los que el Gobierno central no contemplaba las necesidades electorales del partido provincial, provocando entonces la protesta de su jefe. En 1903 Ybarra se quejaba de esto a Maura: :Ustedes no dan ninguna clase de facilidades... No es mucho pedir que de cien pueblos se obtenga uno, a cambio de las facilidades que esto presta a los amigos del Gobierno. ${ }^{10}$,

De todas maneras, normalmente, el correcto funcionamiento del sistema político de la Restauración permitió el desarrollo consensuado de este mecanismo, e incluso el líder liberal sevillano, Rodríguez de la Borbolla, dirigió a Dato, como Ministro de Gobernación, peticiones de esta clase: en 1900 , por ejemplo, le escribía pidiéndole que telegrafiase al Gobernador Civil para que tenga en cuenta mis indicaciones en el nombramiento de concejales del pueblo de Coria del Rio como ya lo hizo usted el año pasado. ${ }^{11}$. En años posteriores, el desmedido uso de este método de presión electoral constituirá una prueba de la progresiva crisis del sistema político de la Restauración. Así, en 1913 y 1916, nos encontramos repetidas quejas de Ybarra a los Ministros de Gobernación sobre la transformación de estos arreglos en "persecuciones" políticas hacia sus amigos ${ }^{12}$.

De igual manera que se recurría a estos particulares amigos políticos en los momentos preelectorales, o incluso con mayor frecuencia, las

(8) Carta del Marqués de San Marcial a Villaverde, 8.10.1900. Archivo Dato, Real Academia de la Historia (Madrid).

(9) Carta de Eduardo Ybarra a Dato, 28.10.1899; cartas de Tomás Ybarra a Dato,8.7.1899 y 20.7.1899. Archivo Dato, Real Academia de la Historia (Madrid). Listado de recomendaciones pendientes por Ministerios: Ministerio de Gobernación (1913). Archivo Ybarra (Sevilla).

(10) Carta de Eduardo Ybarra a Maura, 19.2.1913. Archivo Maura, Fundación Maura (Madrid).

(11) Carta de Rodríguez de la Borbolla a Dato, 16.10.1900. Archivo Dato, Real Academia de la Historia (Madrid).

(12) Carta de Sánchez Guerra, Ministro de Gobernación a Tomás Ybarra, 24.11.1913. Carta de Dato a Tomás Ybarra, 12.9.1916, incluyendo una del Ministro de Gobernación. Archivo Ybarra (Sevilla). 
peticiones de intervención se renovaban cuando la Comisión de Actas o el Tribunal Supremo procedian al examen de las actas problemáticas. Los principales políticos sevillanos, Borbolla e Ybarra, e incluso otros de menor entidad, presionaban a favor de la aprobación o anulación de algunas actas o de elecciones enteras, como refleja la correspondencia privada. En 1899, por ejemplo, Eduardo Ybarra escribía, dando instrucciones en este sentido, a su hermano Tomás, quien se encontraba en Madrid gestionando la aprobación de las elecciones municipales de Sevilla capital: :...deseo que se acabe de una vez lo de las elecciones. Si no se pueden aprobar forzando la máquina todos, sí el mayor número de distritos ${ }^{13}$. De nuevo, la utilización de este recurso a favor del pacto entre liberales y conservadores es prueba del buen funcionamiento del sistema de la Restauración en Sevilla: al igual que Borbolla recurría a Dato en solicitud de este tipo de favores, Ybarra lo hacía a Ministros liberales. En una ocasión, Ybarra se empeñó personalmente en la dēfensa del acta de un liberal que había salido elegido con el apoyo conservador, gestionando su pretensión con éxito ${ }^{14}$.

Pero, tal como anunciábamos, la ayuda de los amigos políticos no sólo es requerida en función del cargo público por ellos ocupado, sino que también puede exigir otras formas de influencia. Ybarra recurrirá a Dato para que, en virtud de su personal vinculación con la compañía de ferrocarriles M.Z.A. (miembro del Consejo de administración), solicite a distintos cargos de la empresa que utilicen su capacidad de presión económica-laboral sobre los empleados de la misma para que éstos voten la candidatura deseada. En 1909, por ejemplo, le escribía para que el ingeniero de las minas de la M.Z.A. en Villanueva del Río thaga de la manera más oportuna y conveniente alguna indicación al personal a sus órdenes para que voten la candidatura conservadora.15.

Más allá de estas ayudas concretas, la vinculación con algún personaje político nacional es fundamental para el político provincial, pues el mantenimiento de su clientela se basa, en muy buena parte, en la concesión de favores relacionados con la administración pública gracias a la recomendación de estos amigos; y ,como veremos en los epígrafes siguientes, la clientela, en sus distintos niveles, constituye el segundo y complementario argumento para el control del proceso electoral.

(13) Carta de Eduardo Ybarra a Tomás Ybarra, 5.7.1899. Archivo Ybarra (Sevilla).

(14) Carta de Rodríguez de la Borbolla a Dato, 21.7.1899: .Uno mi ruego al que ya le habrá hecho D.Eduardo Ybarra../ Carta del Ministro de Gobernación a Tomás Ybarra, 15.6.1916. Archivo Ybarra (Sevilla)./Carta de Eduardo Ybarra a Maura, 16.1.1906. Archivo Maura, Fundación Maura (Madrid).

(15) Carta de Eduardo Ybarra a Dato,26.9.1909. Archivo Dato, Real Academia de la Historia (Madrid). 


\section{LOS CLIENTES SITUADOS EN LA ADMINISTRACION DE JUSTICIA}

Los amigos o dependientes situados en la administración de justicia constituyen el primer nivel de la clientela, en cuanto a efectividad, en su faceta de instrumento electoral. La existencia de este tipo de vinculaciones es característica de la Restauración, pues la utilización de medios judiciales en la ejecución de arreglos electorales slegaliza* tales procedimientos.

Analicemos, en primer lugar, sobre qué realidades se origina la vinculación-dependencia entre el político y el juez. En la misma época de la Restauración, existió clara conciencia de los fundamentos de tal vinculación. Con toda su carga de crítica regeneracionista, Ramón Pérez de Ayala escribía en 1917 "El factor jurídico*, apuntando el origen de la dependencia del poder judicial respecto al político: el nombramiento de estos cargos depende, más o menos directamente, del ejecutivo. Por un lado, cada vez que un partido accede al poder, el nuevo Gobierno organiza a su imagen y semejanza el listado de jueces municipales, y como rla vida en las aldeas, villas y poblaciones depende en gran parte, sobre todo la de las aldeas y villas, de los arbitrios del juez municipal...teniendo en la mano al juez municipal, las elecciones están casi ganadas". Por otro lado, "si bien los gobernantes no nombran jueces de primera instancia, que son embriones de magistrado, gozan luego de la libertad de otorgarles ascensos y disponer traslados ${ }^{16}$. En definitiva, entre el juez, que consigue sus puestos o ascensos gracias a la intervención discrecional del político, y éste, se establecerả una relación de reciprocidad en el favor, en este caso más vinculante que la analizada anteriormente (punto 1).

El estudio de un caso concreto $^{17}$, como el que proponemos en nuestra comunicación, corrobora y especifica el doble origen de esta dependencia. El nombramiento de los jueces municipales aparece claramente determinado por la decisión del político provincial que dirige al partido en el poder. La designación de un plantel de jueces afines era una exigencia en primer lugar, por su efectividad electoral, de los caciques locales y de los directores de distritos, quienes solían apurar esta fácil fórmula de control. Sirva de ejemplo la carta escrita por Eduardo Ybarra a Dato en 1903: "Se acerca la época de la renovación bienal de jueces

(16) R.PEREZ DE AYALA: Escritos políticos. Madrid. Alianza. 1967. pP 73-82.

(17) S.CRUZ ARTACHO realiza un análisis del poder judicial en Granada partiendo de una fuente tan novedosa como el Archivo de la Audiencia Territorial: .La Administración de Justicia en Granada durante el primer tercio del siglo XX. La acción de los jueces municipales., Espacio, Tiempo y Forma (Madrid), №3 Serie V (Octubre 1990), pp173189.

(18) Carta de Eduardo Ybarra a Dato, 12.1.1903. Archivo Dato, Real Academia de la Historia (Madrid). 
municipales; los amigos desean, como es natural, ocupar los cargos....18. Con este tipo de indicaciones, más las propias limitaciones y necesidades, el jefe provincial del partido efectuaba listados (una especie de pequeño *encasillado *) con los nombramientos deseados ${ }^{19}$, que en manos del Gobernador Civil llegaban a materializarse si el Presidente de la Audiencia -como solía ocurrir- no planteaba especiales dificultades. En caso de presentarse algún problema, se recurría a la intervención del poder central, ya que este primer paso en el control de la administración de justicia era prioritario para el funcionamiento *armónicon del sistema: una vez garantizada esta instancia de influencia, no suele ser necesario recurrir a ulteriores medios de presión electoral. Por ello, el nombramiento para estos puestos de amigos y dependientes podía convertirse en un motivo de enfrentamiento interno entre las distintas facciones de un partido, con el consiguiente entrecruzamiento de recomendaciones ${ }^{20}$.

Por otro lado, la inclusión de jueces, fisccales, abogados, etc, en la clientela privada del cacique, a través de la concesión de favores particulares, completaba el dominio privado sobre este sector de la administración pública. El tipo de favor que con más frecuencia vinculaba decisivamente era la gestión de un puesto de trabajo, ascenso o traslado. La correspondencia entre Ybarra y los sucesivos Ministros de Gracia y Justicia recoge constantes peticiones en este sentido, afectando incluso a puestos tan decisivos como los de magistrados y presidentes de distintas Audiencias Provinciales o Territoriales. En 1903, por ejemplo, Dato recibía una carta de Ybarra en la que se recomendaba el ascenso a presidente de sala de un magistrado de la Audiencia sevillana, F.Pozzi, en el que tenían gran interés tanto Ybarra como $\cdot$ los amigos todos. ${ }^{21}$. En pocos años, Pozzi acabará siendo Presidente de la Audiencia de Sevilla. La gestión de este tipo de favores se completaba con mediaciones de otra índole: lo mismo encontramos a Ybarra proponiendo en el Senado una enmienda en el presupuesto de Gracia y Justicia para que fuese incluida una bonificación de 1500 ptas. en el sueldo de magistrados y jueces de primera instancia, que siendo requerido por el presidente de la Audiencia de Sevilla para que con su influencia agilizase la concesión de dinero para el acondicionamiento final y la adquisición de mobiliario para el recién acabado Palacio de Justicia ${ }^{22}$.

(19) Listado .Para jueces municipales. (1913-1914). Archivo Ybarra (Sevilla).

(20) Carta de Eduardo Ybarra a Maura, 21.3.1909, informándole de la existencia de varios candidatos para el puesto de juez municipal en un pueblo complicado políticamente. Archivo Maura, Fundación Maura (Madrid).

(21) Carta de Eduardo Ybarra a Dato, 21.1.1903. Archivo Dato, Real Academia de la Historia (Madrid).

(22) Sevilla, 12.12.1908 y 13.2.1909. Hemeroteca Ybarra (Sevilla). 
El amplio control sobre la administración de justicia que estas vinculaciones otorgaban al gestionador de favores, le permitía contar con un importante instrumento de organización electoral, que, como ya hemos comentado, recubría los distintos manejos con una superficial pero suficiente apariencia de legalidad. Cuando llegaban los momentos electorales, el político recurría a estos amigos y clientes esperando la devolución del favor, fundamentalmente a través de su capacidad de abrir procesos judiciales contra las corporaciones municipales dominadas por la oposición, para dejar campo libre al nombramiento de interinos afines. De la eficacia electoral de la clientela de un político provincial como Ybarra es buena prueba el hecho de que incluso Maura recurriese a ella en alguna ocasión: "Me dicen que tiene usted amistad con D.Antonio Villanueva, Presidente de la Audiencia de Córdoba, de cuya rectitud tengo las mejores referencias. Pero la furia personal y capricho de cierto personaje contra la candidatura del Sr.Sánchez Guerra en Cabra, mueve ahora todos los resortes para obtener el procesamiento de concejales...,agradecería mucho que pudiese usted fortalecer con su recomendación la resistencia del Sr.Villanueva a las tropelías....23.

Evidentemente, la apertura de procesos de este tipo requiere una justificación, aunque sea formal; es decir, la existencia de algún delito imputable a concejales y alcaldes, algo no muy difícil dado el crítico estado de la administración municipal durante la Restauración ${ }^{24}$. El uso político de estas irregularidades queda puesto de manifiesto en los momentos preelectorales, cuando los políticos reúnen todos los argumentos utilizables en la apertura de procesos contra los ayuntamientos que se necesita "remover", siendo las acusaciones más frecuentes las relacionadas con la hacienda local ${ }^{25}$.

La aplastante eficacia del mecanismo puede inducir a los políticos más prepotentes a un uso abusivo del mismo, en detrimento del correcto funcionamiento del sistema. Este fue el caso de Borbolla, jefe del partido liberal sevillano y árbitro indiscutible de la vida política provincial desde comienzos de siglo. Así se refleja en una carta escrita por Ybarra a Dato, describiéndole el último recurso puesto en práctica por Borbolla para presionar a los jueces de la provincia: “Ahora, para cohibir a los jueces se ha inventado un procedimiento, tratándose de funcionario que esté próximo a ascender y por tanto traslado que impone subir de categoría. Aquí se da el caso de haber mandado un mapa de España donde se señalan

(23) Carta de Maura a Tomás Ybarra, 26.4.1901. Archivo Ybarra (Sevilla).

(24) J. TUSELL: Oligarquía y caciquismo en Andalucía, (1890-1923). Barcelona. Planeta. 1976. pp 75-99 (.El encasillado a nivel local.).

(25) Provincia de Sevilla. Impuesto de Consumos. Débitos de los ayuntamientos en 30 Septiembre 1908. Archivo Ybarra (Sevilla). 
los juzgados inmediatos a su actual residencia si procesa y en el mismo plano los juzgados lejanos que más perjuicio pueda causarles si no procesa." Le informaba asimismo de que era el apoyo del Gobernador Civil (que .es una estampilla de Borbolla y su gente.) lo que le había permitido llevar a la práctica este procedimiento, a pesar del disgusto del mismo Presidente de Gobierno, consciente -como Ybarra en parte- de la peligrosa disfunción que para el sistema del pacto constituía este tipo de comportamientos: "Seguimos aquí bajo el yugo de un gobernador sin interés por el prestigio del cargo, del propio ni del Gobierno. Este se escandaliza de lo que hace. Moret afirma que anulará todo lo hecho, que no concibe cómo se hacen esas cosas... ${ }^{26}$.

Aunque el procesamiento de las corporaciones municipales contrarias que se resistían a ceder el poder suele ser la fórmula básica de utilización de la clientela judicial, no podemos dejar de mencionar otro tipo de funcionalidad electoral constatada a través de la documentación privada. Nos referimos a la recomendación del cierre de causas judiciales seguidas contra individuos por delitos electorales. En 1908 Tomás Ybarra solicitaba al Presidente de la Audiencia sevillana vvea si es posible acordar el sobreseimiento de una causa abierta por este motivo, dado que el acusador no iba a acudir a la vista ${ }^{27}$.

\section{OTROS CLIENTES PARTICULARES}

Aunque el conjunto de los clientes situados en la administración de justicia sea el sector más importante de la clientela de un cacique en su faceta de instrumento de control electoral, el resto del grupo cumple también funciones en este mismo campo. Su papel puede ser menos decisivo, dada la aplastante eficacia del componente judicial, y su comportamiento más difícil de tipificar; pero de hecho el cacique, cabeza rectora de una clientela, recurre en la medida de sus posibilidades a estos otros elementos, encomendándoles distintas misiones electorales que acaban completando su control sobre el proceso. Prueba de ello lo constituye el hecho de que la posesión de una clientela particular sea un argumento de peso frente al poder central, cara a conseguir el apoyo oficial frente a otros candidatos: el tener elementos propios", "numerosas fuerzas", etc, en el distrito al que se aspira son las principales razones alega das para obtener el encasillamiento ${ }^{28}$.

(26) Carta de Eduardo Ybarra a Dato (S.F.) Archivo Dato, Real Academia de la Historia (Madrid).

(27) Carta de Tomás Ybarra al presidente de la Audiencia de Sevilla, F.Pozzi. 4.2.1908. Archivo Ybarra Sevilla.

(28) Carta de Eduardo Y'úarra a Maura, 6.3.1907. Archivo Maura, Fundación Maura (Madrid). Carta de Cristóbal Puerta a Dato, 9.1.1902. Archivo Dato, Real Academia de la Historia (Madrid). 
El recurso por parte del cacique a su clientela en momentos electorales se fundamenta en la obligación de ésta respecto al patrono, en relación a lazos de dependencia y gratitud derivados de la concesión de favores de muy diversa índole. En el caso concreto de Ybarra, hemos podido reconstruir una amplia clientela originada tanto en la capacidad de gestión del patrono frente a la administración pública -la concesión de puestos de trabajo en las distintas ramas de la administración, la gestión de ascensos y traslados, la solución a favor del cliente de relaciones conflictivas con la administración (multas, procesos judiciales, quintas, concesión de títulos...)- como en la capacidad de dispensar estos mismos beneficios basándose en su propio status económico -un puesto de trabajo en empresas del grupo, una ayuda en dinero, el pago de una carrera, etc- ${ }^{29}$. Dentro de este amplio campo clientelar nos interesa apuntar algunos sectores concretos que pueden tener especial interés para su instrumentalización electoral. Nos referimos a clientes instalados en las instituciones y administraciones que intervienen en la preparación y materialización del encasillado. El Gobierno Civil de la provincia es una de estas instancias, y en ella encuentran su sitio varios elementos de la clientela analizada: Oficiales, Auxiliares, e incluso militares colaboradores. Sirva como muestra la recomendación hecha por Ybarra a Maura para que evitase el traslado de un Teniente Coronel de Sevilla, por cuanto éste había sido *un buen auxiliar de los Gobernadores Civiles que aquí ha habido", motivo por el que en el asunto se interesan varios amigos: ${ }^{30}$. Similares gestiones colocan a clientes en la Diputación Provincial, la Delegación de Hacienda, el servicio de Correos y las distintas fuerzas de orden público.

Como ya mencionábamos, no resulta fácil explicar la funcionalidad electoral de estos elementos clientelares, por la escasa unidad constatable en sus actuaciones; pero en líneas generales podríamos distinguir dos modos de intervención electoral, que, por otra parte, confirman el distinto comportamiento político de los ámbitos urbano y rural. En los pueblos se puede rastrear la efectividad electoral de clientes que basan su intervención en su particular status socio-profesional y la influencia de él derivada. En la ciudad estos elementos clientelares basan su funcionalidad electoral en el trabajo directo de los distritos.

En el primer sentido, ¿cuáles son los elementos con influencia transformable en efectividad electoral? Evidentemente, en primer lugar en cuanto a su eficacia, los clientes situados en los ayuntamientos, quienes desde esta instancia de poder controlan con facilidad el proceso electo-

(29) SIERRA ALONSO,M: La familia Ybarra, empresarias y políticos. Sevilla. Muñoz Moya. 1992.

(30) Carta de Eduardo Ybarra a Maura. Archivo Maura, Fundación Maura (Madrid). 
ral. Concejales, secretarios y alcaldes se incluyen en clientelas privadas a título personal, y su intervención en los procesos electorales facilita enormemente la labor del cacique. En 1913, por ejemplo, Tomás Ybarra recibía una carta de su hijo en la que le informaba acerca de la marcha de los trabajos preparatorios de las próximas elecciones provinciales: $\cdot$ Mejías ha llamado a los de los pueblos y hasta ahora responden muy bien... pues Almansilla nos da todo lo que pueda, Brenes opina Mejías que está mejor de lo que se creía, y Gelves resulta que el capataz de Narciso [Narciso Ciaurriz, candidato del partido] es el Alcalde. ${ }^{31}$. Pero no sólo los clientes que controlan el ayuntamiento pueden prestar su apoyo llegado el momento electoral; también figuran en este grupo personajes como el médico o el maestro del pueblo, debido a su capacidad de influencia sobre la vida local, y de hecho los políticos buscan sus adhesiones y defienden sus intereses ${ }^{32}$. El comportamiento de esos elementos es vigilado por sus patronos, para controlar el cumplimiento de la reciprocidad clientelar. A propósito de las elecciones provinciales de 1913 antes citadas Tomás Ybarra recibía la siguiente carta de un sobrino: ‘De las cosas del domingo [día en el que se celebraron las elecciones] supongo que Tomás te habrá escrito y sabrás de la conducta del médico de la Puebla y del secretario de Tomares.33.

La mayor movilización política urbana invalida parcialmente esta fórmula de instrumentalización electoral de los clientes, y aquí los dependientes son requeridos más por su capacidad de trabajo que por su significación personal. Los clientes son solicitados fundamentalmente para trabajar comó interventores en los distintos distritos electorales. En 1905 Eduardo Ybarra preparaba las elecciones municipales en Sevilla capital, y escribía a su hermano Tomás dándole instrucciones sobre el asunto: ‘...sobre todo hay que revisar los interventores. Para el desempeño de esta función lo mismo valen curas, que sacristanes que empleados del matadero: $\cdot \mathrm{El} 10^{\circ}$ [distrito] ...comprende San Roque, a cuyo cura le hablé yo y está dispuesto a trabajar... El antiguo sacristán Ortiz, que era de San Roque, actual empleado del Ayuntamiento en el matadero, conviene que trabaje aunque no muy ostensiblemente. Este tenía otra sección, y si lo ven los republicanos le pueden hacer daño. ${ }^{34}$. Los clientes también pueden realizar eficaces trabajos electorales encargándose de la compra de votos.

(31) Carta de Tomás Ybarra Lasso de la Vega a Tomás Ybarra, 24.9.1913. Archivo Ybarra (Sevilla).

(32) Carta de Eduardo Ybarra a Tomás Ybarra (1898). Archivo Ybarra (Sevilla). El Noticiero Sevillano, 30.9.1916. Hemeroteca Municipal de Sevilla.

(33) Carta de Luis Ybarra a Tomás Ybarra, 14.10.1913. Archivo Ybarra (Sevilla).

(34) Carta de Eduardo Ybarra a Tomás Ybarra, 17.10.1905. Archivo Ybarra (Sevilla). 
Indicio del distinto nivel de desarrollo político en el que se encuentra la ciudad frente al campo lo constituye el hecho de que este tipo de apoyos no siempre se deban a clientes obligados por la concesión de favores, sino que a veces procedan de personas con una vinculación menos intensa y duradera con el cacique, a las que se compra el apoyo. Hemos encontrado en el archivo Ybarra las cuentas de los gastos realizados durante unas elecciones municipales, documento excepcional por lo difícil de su conservación. Entre otras partidas de gastos figuran los siguientes conceptos: :.... a un interventor de la $6^{2}, \ldots$ a dos hombres que trabajaron en los Distritos $10^{\circ}$ y $5^{\circ}, \ldots$ al individuo que ayudó a fijar carteles y votó en el $5^{\circ}$ distrito....35.

Concluiremos este apartado sobre la utilización de la clientela en el ámbito urbano comentando un último ejemplo de intervención con consecuencias electorales. Las instituciones que por derecho propio eligen a un representante en el Senado, en concreto la Universidad y la Sociedad Económica de Amigos del País, son también solar de enfrentamiento entre los distintos políticos provinciales, y para su control se pueden utilizar instrumentos parecidos a los argumentados en otras instancias de poder: la amistad personal y la dependencia clientelar. Estas instituciones votan una candidatura u otra según la adscripción de los miembros de sus juntas directivas, y por ello el control del nombramiento de estos cargos se convierte en un nuevo motivo de movilización política. En 1905 , por ejemplo, cuando el partido liberal acababa de acceder al poder, debía procederse a la renovación de los principales cargos de la Sociedad Económica de Amigos del País: el Presidente y el Secretario General. Los conservadores, que habían controlado estos puestos, se resistían a cederlos. Francisco González Alvarez, miembro del partido y de la Sociedad Económica, preparaba con los Ybarra la manera de conseguir que tales puestos "queden de los conservadores". "Para esto se hace necesario presentar a la $1^{2}$ Junta general el día 19 la mayor cantidad de socios posibles...* con objeto de que tengan derecho a voto en el momento de elegir los cargos de la junta directiva. En la carta de donde proceden estos comentarios se incluye un listado con los posibles nuevos socios que hay que "trabajar. En él, además de figurar amigos que están por encima de la relación clientelar (José Piñar y Pickman, Tomás Osborne), aparecen nombres de dependientes ("Adolfo Meyer, vuestro sastre") que ayudarían a controlar con éxito la elección ${ }^{36}$.

(35) Cuenta de pactos en las elecciones.... Archivo Ybarra (Sevilla).

(36) Carta de Francisco González Alvarez a Tomás Ybarra, (Octubre.1905). Archivo Ybarra (Sevilla) 


\section{LOS CLIENTES COLECTIVOS}

En el caso de los políticos profesionales, el núcleo de la clientela solía verse rodeado y completado por un círculo más amplio de beneficiarios de su gestión. Este círculo estaba compuesto por grupos de diversa naturaleza: pueblos, ciudades, instituciones, asociaciones económicas, profesionales o culturales... Eran clientes colectivos que se incluían en la relación de patronazgo de manera conjunta, no a través de los lazos individuales de obligación que vinculaban a los componentes del núcleo clientelar. El apoyo prestado por este grupo a su patrocinador constituyó un importante complemento en la conformación del protagonismo político de éste. Tal respaldo, como el de los otros sectores clientelares ya analizados, también tuvo una lectura electoral, pues cumplió funciones concretas de apoyo en este sentido.

Son fundamentalmente dos los sujetos colectivos relacionados con el cacique a través del favor que luego podían devolverle su apoyo en las elecciones: las localidades rurales cuyas oligarquías se vinculaban a un cacique de rango provincial y las asociaciones económicas o profesionales que recurrían al cacique como mediador ante el poder central de sus intereses de grupo. Cada una de estas relaciones tiene fundamentos y manifestaciones electorales diferentes, a pesar de compartir ese carácter común de .clientes colectivos. La base del apoyo de los dos colectivos la constituye la devolución del favor concedido por el cacique, pero la obligación consecuente del favor es mucho más estrecha en el caso de los pueblos y más hipotética en el de los grupos económicos-profesionales. En consecuencia, el apoyo electoral prestado por cada grupo será muy distinto.

La vinculación entre los pueblos y el cacique provincial se realizaba a través de la iniciativa de las respectivas oligarquías locales. En 1887, por ejemplo, el órgano de prensa conservador celebraba la entrada en el partido de un individuo de Bollullos, Pérez Merchante, "con sus 500 contribuyentes y electores del distrito de la Palma ${ }^{37}$, entrada seguramente debida a la vinculación de este sujeto con algún primate del comité provincial del partido: Las oligarquías locales, dada la desconexión existente entre el poder central y las realidades locales, necesitaban recurrir al cacique provincial como una figura mediadora que, gracias a sus contactos con la administración pública y a su amistad personal con los miembros del gobierno central, era capaz de gestionar con éxito las

(37) El Universal, 15.7.1887. Hemeroteca Ybarra (Sevilla). 
demandas locales ${ }^{38}$. Las peticiones realizadas eran muy concretas (la construcción de carreteras, la concesión de ayudas económicas con motivo de inundaciones, la resolución de conflictos sobre términos municipales, etc. ${ }^{39}$ ), pero su resolución positiva vinculaba decisivamente al solicitante con el gestor, con una lectura electoral muy eficaz, como veremos posteriormente. Ya antes (3) habíamos analizado la vinculación entre miembros de las oligarquías locales y caciques provinciales desde el punto de vista del cliente individual: el alcalde, el secretario del ayuntamiento, el médico, etc. Pero ahora estamos contemplando a este mismo cliente desde su significado colectivo, como cabeza rectora a su vez de una clientela menor a la que inscribe, para su mantenimiento, en el grupo superior del cacique provincial, conformando la estructura de poder piramidal característica de la Restauración.

También las agrupaciones económicas y profesionales recurrieron a los caciques provinciales como gestores de sus intereses ante el poder central: Liga de Contribuyentes, Cámara de Comercio, Liga Agraria, Colegio de Farmacéuticos..., son algunos ejemplos de las asociaciones sevillanas que solicitaron el poder gestor de Ybarra para así presentar mejor sus reivindicaciones ante el Gobierno ${ }^{40}$. En compensación, el político pretendía pulsar la gratitud de estos grupos en los momentos electorales, pero el carácter colectivo del cliente y la peculiar independencia económica derivada de su naturaleza disolvían el lazo de obligación, por lo que, normalmente, la reciprocidad en el favor quedaba reducida a una simple predisposición favorable.

Por el contrario, la devolución del favor en forma de ayuda electoral por parte de los pueblos resultaba de capital importancia para el político, que por ello controlaba y daba instrucciones a este sector clientelar en los momentos preelectorales. La devolución del favor en este caso tiene

(38) Respecto a la figura del buen cacique afirma ROMERO MAURA: -...hay que huir por encima de todo de la creencia, $\tan$ constante en la literatura anticaciquista, de que el caciquismo sólo beneficiaba al cacique y a un grupo reducido de allegados suyos. La figura del cacique cuyo día se consume principalmente en ir de un ministerio a otro, de un negociado al siguiente, para obtener todo lo que le reclaman los protegidos y el distrito, es por lo menos tan típica como la del doctor en atropellos. Op.cit, p 82.

(39) Carta de Ignacio Villalón, cacique conservador de Morón, a Tomás Ybarra,13.10.1987: -...me apresuro a dar a usted las gracias por su gestión en el asunto que le recomendé de la catretera./ Carta de Dato a Tomás Ybarra, 24.3.1899: ....he recibido su carta en que me recomienda se conceda alguna cantidad para socorrer al pueblo de Herrera. Archivo Ybarra (Sevilla)./ Carta de Tomás Ybarra a Dato, 29.8.1900: recomendándole una petición del Ayuntamiento de Lora de Estepa para que se fijen los límites de su término municipal respecto a Estepa y Casariche. Archivo Dato, Real Academia de la Historia (Madrid).

(40) SIERRA ALONSO,M: La familia Ybarra... 
una simple y eficaz versión electoral: la oligarquía local, que ha situado a su comunidad bajo la protección del cacique provincial, *vacía a su favor el censo electoral de la localidad en las actas oficiales. La desmovilización política, el consenso social establecido en torno al mecanismo del favor y el control de los ayuntamientos por las oligarquías locales posibilitaban esta fórmula de arreglo electoral. Por ello, la existencia de este tipo de vinculaciones entre un político y una localidad era una baza de triunfo electoral reconocida y admitida abiertamente en la época: con motivo de las elecciones generales de 1901, el mismo órgano borbollista, El Porvenir, explicaba el triunfo conservador en la localidad de Dos Hermanas por la influencia de un buen cacique como Tomás Ybarra ‘a quien todos quieren y estiman en este pueblo. En unos bosquejos biográficos que el mismo periódico publicaba al día siguiente sobre los candidatos a diputados, Tomás Ybarra aparecía descrito como un defensor de los intereses de la provincia, que gestionaba constantemente con éxito gracias a su "grandísima influencia en las regiones oficiales. ${ }^{41}$.

En cambio, como ya adelantábamos, el apoyo de esos otros colectivos económicos-profesionales era mucho más aleatorio, lo cual no fue óbice para que en las ocasiones electorales los políticos procurasen recabar su auxilio. En 1893, por ejemplo, la prensa conservadora intentaba recurrir al sector comercial sevillano para que prestase su apoyo a la candidatura de Eduardo Ybarra, a quien presentaba como un thijo del Comercio que conoce perfectamente los problemas económicos", a la vez que recordaba sus gestiones a favor del sector ${ }^{42}$. De todas maneras, a veces, el apoyo era más práctico: en 1908 Eduardo Ybarra escribía a su hermano Tomás, que se encontraba en Madrid, recomendándole gestionase una solicitud de los taberneros sevillanos. "Preséntala al Ministro con gran recomendación, porque...éstos nos van a dar sus votos. ${ }^{43}$.

En definitiva, creemos haber mostrado cómo dos tipos de círculos de relaciones fundamentadas en el favor -los amigos políticos y la clientela- son instrumentalizadas por el cacique de ámbito provincial para conseguir el control de los procesos electorales. En Sevilla el recurso a estos mecanismos se desarrolla sin dificultad durante el grueso cronológico de la Restauración. Cuando empiecen a apreciarse disfunciones en su aplicación (sobre todo a partir de la segunda década del siglo $\mathrm{XX}$ ) se deberá a la crisis del espíritu y de la práctica del pacto político en el contexto de la crisis general del sistema. Desde luego, esta manifestación electoral no es la única función política que adquiere el mecanismo

(41) El Porvenir, 23.5.1901 y 24.5.1901. Hemeroteca Municipal de Sevilla.

(42) El Universal, 1.3.1893. Hemeroteca Ybarra.

(43) Carta de Eduardo Ybarra a Tomás Ybarra (1908). Archivo Ybarra (Sevilla). 
del favor durante la Restauración. Jefaturas y otros cargos, adscripciones a los partidos, divisiones en facciones..., son fenómenos políticos que en la Restauración se explican, en buena parte, en relación al favor. Estos comportamientos políticos, que escapan a las posibilidades de esta comunicación y abordaremos en otro estudio, convierten a los partidos de la Restauración en grupos clientelares. Clientelas políticas con distintos componentes y distintas dependencias que las clientelas particulares a las que hemos hecho referencia, pero, al fin y al cabo, clientelas con el mismo fundamento: el favor. 